\title{
Ground states and high energy solutions of the planar Schrödinger-Poisson system
}

\author{
Miao $\mathrm{Du}^{\mathrm{a}}$, Tobias Weth ${ }^{\mathrm{b}, *}$ \\ a School of Mathematical Sciences, Nanjing Normal University, \\ Nanjing 210023, P.R. China \\ b Institut für Mathematik, Goethe-Universität Frankfurt, \\ D-60629 Frankfurt am Main, Germany \\ $\|$
}

\begin{abstract}
In this paper, we are concerned with the Schrödinger-Poisson system

$$
\begin{cases}-\Delta u+u+\phi u=|u|^{p-2} u & \text { in } \mathbb{R}^{d} \\ \Delta \phi=u^{2} & \text { in } \mathbb{R}^{d}\end{cases}
$$

Due to its relevance in physics, the system has been extensively studied and is quite well understood in the case $d \geq 3$. In contrast, much less information is available in the planar case $d=2$ which is the focus of the present paper. It has been observed by Cingolani and the second author [6] that the variational structure of (0.1) differs substantially in the case $d=2$ and leads to a richer structure of the set of solutions. However, the variational approach of [6] is restricted to the case $p \geq 4$ which excludes some physically relevant exponents. In the present paper, we remove this unpleasant restriction and explore the more complicated underlying functional geometry in the case $2<p<4$ with a different variational approach.
\end{abstract}

MSC: 35J50; 35Q40

Keywords: Schrödinger-Poisson system; Logarithmic convolution potential; Ground state solutions; Variational methods

\section{Introduction}

The present paper is devoted to standing (or solitary) wave solutions of Schrödinger-Poisson systems of the type

$$
\begin{cases}i \psi_{t}-\Delta \psi+W(x) \psi+m \phi \psi=|\psi|^{p-2} \psi & \text { in } \mathbb{R}^{d} \times \mathbb{R} \\ \Delta \phi=|\psi|^{2} & \text { in } \mathbb{R}^{d}\end{cases}
$$

where $\psi: \mathbb{R}^{d} \times \mathbb{R} \rightarrow \mathbb{C}$ is the time-dependent wave function, $W: \mathbb{R}^{d} \rightarrow \mathbb{R}$ is a real external potential, $m \in \mathbb{R}$ is a parameter and $2<p<2^{*}$. Here, $2^{*}$ is the so-called critical Sobolev exponent, that is, $2^{*}=\frac{2 d}{d-2}$ in case $d \geq 3$ and $2^{*}=\infty$ in case $d=1,2$. Evolution problems of the type (1.1) arise in many problems from physics. We refer the reader e.g. to [16], where (1.1) is discussed in a quantum mechanical context where the particular exponent $p=2+\frac{2}{d}$ appears in the case $d \leq 3$,

\footnotetext{
* Corresponding author

E-mail addresses: dumiaomath@163.com, weth@math.uni-frankfurt.de.
} 
see [16, p. 761]. The function $\phi$ represents an internal potential for a nonlocal self-interaction of the wave function $\psi$. The usual ansatz

$$
\psi(x, t)=e^{-i \omega t} u(x) \quad \text { with } \omega \in \mathbb{R}
$$

for standing wave solutions of (1.1) leads to the Schrödinger-Poisson system

$$
\begin{cases}-\Delta u+V(x) u+m \phi u=|u|^{p-2} u & \text { in } \mathbb{R}^{d}, \\ \Delta \phi=u^{2} & \text { in } \mathbb{R}^{d},\end{cases}
$$

where $V(x)=W(x)+\omega$. The second equation in (1.2) determines $\phi: \mathbb{R}^{d} \rightarrow \mathbb{R}$ only up to harmonic functions. It is natural to choose $\phi$ as the negative Newton potential of $u^{2}$, that is, the convolution of $u^{2}$ with the fundamental solution $\Phi_{d}$ of the Laplacian, which is given by $\Phi_{d}(x)=-\frac{1}{d(d-2) \omega_{d}}|x|^{2-d}$ in case $d \geq 3$ and $\Phi_{d}(x)=\frac{1}{2 \pi} \log |x|$ in case $d=2$. Here $\omega_{d}$ denotes the volume of the unit ball in $\mathbb{R}^{d}$. With this formal inversion of the second equation in (1.2), we obtain the integro-differential equation

$$
-\Delta u+V(x) u+m\left(\Phi_{d} *|u|^{2}\right) u=|u|^{p-2} u \text { in } \mathbb{R}^{d} .
$$

In the three dimensional case, equation (1.3) and its generalizations have been widely studied in recent years, whereas existence, nonexistence and multiplicity results have been obtained under variant assumptions on $V$ and $f$ via variational methods, see e.g. [1 $-3,5,8,8,10,20,24,26,27]$ and the references therein.

We note that, at least formally, (1.3) has a variational structure related to the energy functional

$$
\begin{aligned}
& u \mapsto I(u)= \\
& \frac{1}{2} \int_{\mathbb{R}^{d}}\left(|\nabla u|^{2}+V(x) u^{2}\right) d x+\frac{m}{4} \int_{\mathbb{R}^{d}} \int_{\mathbb{R}^{d}} \Phi_{d}(|x-y|) u^{2}(x) u^{2}(y) d x d y-\frac{1}{p} \int_{\mathbb{R}^{d}}|u|^{p} d x .
\end{aligned}
$$

However, in the case where $d=2$ and $\Phi_{d}(x)=\frac{1}{2 \pi} \log |x|$, the functional $I$ is not well-defined on $H^{1}\left(\mathbb{R}^{2}\right)$, and this is one of the reasons why much less is known in the planar case. Inspired by Stubbe [21, Cingolani and Weth [6] developed a variational framework for the equation

$$
-\Delta u+V(x) u+\left(\log (|\cdot|) *|u|^{2}\right) u=b|u|^{p-2} u \quad \text { in } \mathbb{R}^{2}
$$

within the smaller Hilbert space

$$
X:=\left\{u \in H^{1}\left(\mathbb{R}^{2}\right): \int_{\mathbb{R}^{2}} \log (1+|x|) u^{2} d x<\infty\right\} .
$$

In this work, a positive function $V \in L^{\infty}\left(\mathbb{R}^{2}\right), b \geq 0$ and $p \geq 4$ are considered. In particular, high energy solutions were detected in [6] in a periodic setting where the corresponding functional is invariant under $\mathbb{Z}^{2}$-translations and therefore fails to satisfy a global Palais-Smale condition. In the case where the external potential $V$ is a positive constant, they also obtained the existence of nonradial solutions which have arbitrarily many nodal domains. The key tool in [6] is a strong compactness condition (modulo translation) for Cerami sequences at arbitrary positive energy levels. Such a property fails to hold in higher space dimensions, and it is also not available in the case where $2<p<4$. More precisely, it is an open question whether general Cerami sequences 
for $I$ in $X$ are bounded in the case $2<p<4$, and therefore no existence results for (1.5) have been available for this case. This gap of information is unpleasant not only from a mathematical point of view but also since, as already remarked above, the case $p=3$ is relevant in 2-dimensional quantum mechanical models, see [16, p. 761].

The purpose of this paper is to fill this gap and to provide a counterpart of the results in [6] in the case where $2<p<4$ and $V$ is a positive constant. In particular, we shall prove the existence of ground state solutions and infinitely many nontrivial sign-changing solutions for (1.5) in this case.

At this point, we wish to point out some difficulties of the variational approach in the space $X$. First, while the functional $I$ is translation invariant, the norm of $X$ is not translation invariant. Second, the quadratic part of $I$ is not coercive on $X$. These difficulties have been overcome in [6], and we will partly follow the approach developed there. The key new difficulty in the case where $2<p<4$ is the competing nature of the local and nonlocal superquadratic terms in the functional $I$ and their different behaviour under scaling transformations. In particular, we note that the nonlinearity $u \mapsto f(u):=|u|^{p-2} u$ with $2<p<4$ does not satisfy the Ambrosetti-Rabinowitz type condition

$$
0<\mu \int_{0}^{u} f(s) d s \leq f(u) u \quad \text { for all } u \neq 0 \text { with some } \mu>4
$$

which would readily imply the boundedness of Palais-Smale sequences. Moreover, the fact that the function $f(s) /|s|^{3}$ is not increasing on $\mathbb{R} \backslash\{0\}$ prevents us from using Nehari manifold and fibering methods as e.g. in [19,22]. Moreover, in contrast to the higher dimensional case, the logarithmic convolution term does not have a definite sign on $X$. As a consequence, the boundedness of Cerami sequences becomes a major difficulty in the variational setting.

To state our main results, we assume that $V$ in (1.2) and (1.4) is constant from now on, and without loss we may assume that $V \equiv 1$. Moreover, we focus on (1.2) in the case $m>0$, and by rescaling we may assume $m=2 \pi$. Consequently, we are dealing with system (1.2), the associated scalar equation

$$
-\Delta u+u+\left(\log (|\cdot|) *|u|^{2}\right) u=|u|^{p-2} u \quad \text { in } \mathbb{R}^{2}
$$

and the associated energy functional $I: X \rightarrow \mathbb{R}$ defined by

$$
I(u)=\frac{1}{2} \int_{\mathbb{R}^{2}}\left(|\nabla u|^{2}+u^{2}\right) d x+\frac{1}{4} \int_{\mathbb{R}^{2}} \int_{\mathbb{R}^{2}} \log (|x-y|) u^{2}(x) u^{2}(y) d x d y-\frac{1}{p} \int_{\mathbb{R}^{2}}|u|^{p} d x .
$$

In the following, by a solution of (1.6) we always mean a weak solution, i.e. a critical point of $I$.

Remark 1.1. It has been proved in [6, Proposition 2.3] that every critical point $u \in X$ of $I$ is a classical solution of class $C^{2}$ satisfying $u(x)=o\left(e^{-\alpha|x|}\right)$ as $|x| \rightarrow \infty$ for any $\alpha>0$. Moreover, the function $x \mapsto w(x)=\int_{\mathbb{R}^{2}} \log |x-y| u^{2}(y) d y$ is of class $C^{3}$ on $\mathbb{R}^{2}$ and satisfies

$$
\Delta w=2 \pi u^{2} \quad \text { in } \mathbb{R}^{2}, \quad w(x)-\log |x| \int_{\mathbb{R}^{2}} u^{2} d y \rightarrow 0 \quad \text { as }|x| \rightarrow \infty .
$$

It has been assumed that $p \geq 4$ in [6], but the proof of [6, Proposition 2.3] carries over to the case $p \geq 2$ without change.

Our first main result is concerned with the existence of mountain pass and ground state solutions. For this we define the mountain pass value

$$
c_{m p}=\inf _{\gamma \in \Gamma} \max _{t \in[0,1]} I(\gamma(t)) \quad \text { with } \quad \Gamma=\{\gamma \in C([0,1], X) \mid \gamma(0)=0, I(\gamma(1))<0\} .
$$


Theorem 1.1. Suppose that $p>2$.

(i) We have $c_{m p}>0$, and equation (1.6) has a solution $u \in X \backslash\{0\}$ with $I(u)=c_{m p}$.

(ii) Equation (1.6) has a ground state solution, i.e., a solution $u \in X \backslash\{0\}$ such that $I(u)$ equals the ground state energy

$$
c_{g}:=\inf \{I(v): v \in X \backslash\{0\} \text { is a solution of (1.6) }\} .
$$

In case $2<p<3$, we do not know if the mountain pass energy $c_{m p}$ coincides with the ground state energy $c_{g}$. In the case where $p \geq 3$, we can derive more information. First, we can identify $c_{g}$ with $c_{m p}$, and we can give a natural characterization of the ground state energy as a constrained minimum and as a simple minimax value. Second, we will see that every ground state solution does not change sign. For this purpose, inspired by [20], we introduce the auxiliary functional $J: X \rightarrow \mathbb{R}$ defined by

$$
\begin{aligned}
J(u) & =\int_{\mathbb{R}^{2}}\left(2|\nabla u|^{2}+|u|^{2}-\frac{2(p-1)}{p}|u|^{p}\right) d x \\
& +\int_{\mathbb{R}^{2}} \int_{\mathbb{R}^{2}} \log (|x-y|) u^{2}(x) u^{2}(y) d x d y-\frac{1}{4}\left(\int_{\mathbb{R}^{2}}|u|^{2} d x\right)^{2},
\end{aligned}
$$

and the set

$$
\mathcal{M}:=\{u \in X \backslash\{0\}: J(u)=0\} .
$$

It then follows in a standard way from a Pohozaev type identity given in Lemma 2.4 below that every solution of (1.6) is contained in $\mathcal{M}$. Consequently, the minimal energy value

$$
c_{\mathcal{M}}:=\inf _{u \in \mathcal{M}} I(u)
$$

on $\mathcal{M}$ satisfies

$$
c_{\mathcal{M}} \leq c_{g} \leq c_{m p}
$$

where the latter inequality follows from Theorem 1.1(i). We also define the minimax value

$$
c_{m m}:=\inf _{u \in X \backslash\{0\}} \sup _{t>0} I\left(u_{t}\right) .
$$

where $u_{t} \in X$ is defined by $u_{t}(x):=t^{2} u(t x)$ for $u \in X, t>0$.

Theorem 1.2. Suppose that $p \geq 3$. Then we have

$$
c_{g}=c_{\mathcal{M}}=c_{m m}=c_{m p}
$$

Moreover, this value is attained by $I$ on $\mathcal{M}$, and every function $u \in \mathcal{M}$ with $I(u)=c_{\mathcal{M}}$ is a (ground state) solution of (1.6) and does not change sign.

For equation (1.3) in case $d \geq 3$ with $m>0$, a result corresponding to Theorem 1.1 is proved in [23, Theorem 3.4]. In this case, the convolution term of the energy functional in (1.4) is negative definite, which is of key importance in the proof in [23]. As remarked above, the convolution term in (1.7) does not have a definite sign. In the planar case where $p \geq 4$, the existence of a ground 
state solution has been proved in [6, Theorem 1.1], where also the existence of infinitely many pairs of solutions is established under more general assumptions. As noted before, the proof in [6] does not carry over to the case $2<p<4$ due to the possible lack of boundedness of Cerami sequences.

It is instructive to relate the exponent $p$ to the presence of saddle point structures of the functional $I$. In the case $p \geq 4$ studied in [6], $I$ has a rather simple saddle point structure with regard to the fibres $\{t u: t>0\} \subset X, u \in X \backslash\{0\}$, see [6, Lemma 2.5]. In particular, this implies that the ground state energy coincides with the minimum of $I$ on the associated Nehari manifold and obeys the simple minimax characterization $c_{g}=\inf _{u \in X \backslash\{0\}} \sup _{t>0} I(t u)$, see [6, Theorem 1.1]. In case $2<p<4$ this property is lost, but for $p \geq 3$ we recover a different saddle point structure with respect to the fibres $\left\{u_{t}: t>0\right\} \subset X, u \in X \backslash\{0\}$, see Lemma 4.2 below. This new saddle point structure provides the basis for the proof of Theorem 1.2. In the case $p \in(2,3)$ we could not find any similar saddle point structure of $I$, and we believe that the more complicated geometry of $I$ in this case is related to particular difficulties regarding the boundedness of Cerami sequences.

Our proof of Theorem [1.1 uses some preliminary tools from [6] and is also inspired by [11,12,17]. First we construct a Cerami sequence $\left(u_{n}\right)_{n}$ at the mountain-pass level $c_{m p}$ with the extra property that $J\left(u_{n}\right) \rightarrow 0$ as $n \rightarrow \infty$. From this extra information, we then deduce the boundedness of $\left(u_{n}\right)_{n}$ in $H^{1}\left(\mathbb{R}^{2}\right)$. In the case $p \geq 3$ this step is not difficult, whereas for $2<p<3$ the argument is more subtle. Once this step is taken, we can follow arguments in [6] to pass to a subsequence which after suitable translation - converges to a nontrivial solution $\tilde{u}$ of (1.6) with $I(\tilde{u})=c_{m p}$. Hence the set $\mathcal{K}$ of nontrivial solutions of (1.6) is nonempty. We then consider a sequence $\left(u_{n}\right)_{n}$ in $\mathcal{K}$ with $I\left(u_{n}\right) \rightarrow c_{g}$ as $n \rightarrow \infty$, and in the same way as before we may then pass to a subsequence which after suitable translation - converges to a nontrivial solution $u$ of (1.6) with $I(u)=c_{g}$.

Our third main result is concerned with a symmetric setting with respect to suitably defined actions of subgroups of the orthogonal group $O(2)$, and it will give rise to the existence of infinitely many nonradial sign-changing solutions of (1.6). We need to introduce some notation. Let $G$ be a closed subgroup of the orthogonal group $O(2)$. Moreover, let $\tau: G \rightarrow\{-1,1\}$ be a group homomorphism. Then the pair $(G, \tau)$ gives rise to a group action of $G$ on $X$ defined by

$$
[A * u](x):=\tau(A) u\left(A^{-1} x\right) \quad \text { for } A \in G, u \in X \text { and } x \in \mathbb{R}^{2} .
$$

The following result is concerned with solutions of (1.6) in the invariant space

$$
X_{G}:=\{u \in X: A * u=u \text { for all } A \in G\} .
$$

Theorem 1.3. Suppose that $p>2$. Let $G, \tau$ be as above, assume that $X_{G} \neq\{0\}$, and let

$$
c_{m p, G}=\inf _{\gamma \in \Gamma_{G}} \max _{t \in[0,1]} I(\gamma(t)) \quad \text { with } \quad \Gamma_{G}=\left\{\gamma \in C\left([0,1], X_{G}\right) \mid \gamma(0)=0, I(\gamma(1))<0\right\} .
$$

(i) We have $c_{m p, G}>0$, and equation (1.6) has a solution $u \in X_{G} \backslash\{0\}$ with $I(u)=c_{m p, G}$.

(ii) Equation (1.6) has a $G$-invariant ground state solution $u \in X_{G} \backslash\{0\}$, i.e., the function $u \in X_{G}$ satisfies (1.6) and

$$
I(u)=\inf \left\{I(v): v \in X_{G} \backslash\{0\} \text { is a solution of }(1.6)\right\} .
$$


We remark that if $\tau$ is nontrivial and $A \in G$ is given with $\tau(A)=-1$, then every $u \in X_{G}$ satisfies $u\left(A^{-1} x\right)=-u(x)$. Consequently, we see that $u$ vanishes on the set $\left\{x \in \mathbb{R}^{2}: A x=x\right\}$ and changes sign in $\mathbb{R}^{2}$ if $u \neq 0$. We also point out that Theorem 1.3 has no analogue yet in the higher dimensional case, i.e. for equation (1.3) in case $d \geq 3$ with $m>0$. This remains an interesting open problem in the case where $\tau$ is nontrivial.

We discuss some examples for $G$ and $\tau$.

Example 1.1. (i) Suppose that $G=O(2)$ and $\tau \equiv 1$. Then the space $X_{G}$ consists of radial functions in $X$. In this case, Theorem 1.3 yields the existence of radial ground state solutions.

(ii) Let $G=\left\{i d,-i d, A_{1}, A_{2}\right\}$, where $A_{i}$ is the reflection at the coordinate hyperplane $\left\{x_{i}=0\right\}$ for $i=1,2$. Moreover, let $\tau: G \rightarrow\{-1,1\}$ be the homomorphism defined by $\tau\left(A_{1}\right)=-1$ and $\tau\left(A_{2}\right)=1$. Then $u \in X_{G}$ if and only if

$$
u\left(-x_{1}, x_{2}\right)=-u\left(x_{1}, x_{2}\right) \quad \text { and } \quad u\left(x_{1},-x_{2}\right)=u\left(x_{1}, x_{2}\right) \quad \text { for all } x=\left(x_{1}, x_{2}\right) \in \mathbb{R}^{2} .
$$

Therefore, Theorem 1.3 yields a $G$-invariant ground state solution of (1.6) such that this solution is odd with respect to the hyperplane $\left\{x_{1}=0\right\}$ and even with respect to the hyperplane $\left\{x_{2}=0\right\}$. We point out that this existence result has no analogue for the seemingly similar nonlinear Schrödinger equation

$$
-\Delta u+u=|u|^{p-2} u, \quad u \in H^{1}\left(\mathbb{R}^{2}\right)
$$

Indeed, by [7], (1.20) does not admit nontrivial solutions which vanish on a hyperplane.

(iii) We assume that, for given $k \in \mathbb{N}$, the subgroup $G$ of $O(2)$ of order $2 k$ generated by the (counter-clockwise) $\frac{\pi}{k}$-rotation

$$
A \in O(2), \quad A x=\left(x_{1} \cos \frac{\pi}{k}-x_{2} \sin \frac{\pi}{k}, x_{1} \sin \frac{\pi}{k}+x_{2} \cos \frac{\pi}{k}\right) \quad \text { for } x=\left(x_{1}, x_{2}\right) \in \mathbb{R}^{2} .
$$

Let $\tau: G \rightarrow\{-1,1\}$ be the homomorphism defined by

$$
\tau\left(A^{j}\right)=(-1)^{j} \quad \text { for } j=1, \cdots, 2 k,
$$

where $A^{j}$ is the $\frac{j \pi}{k}$-rotation. Then Theorem 1.3 applies and yields a $G$-invariant ground state solution. Note that any such solution is sign-changing and nonradial.

From Theorem 1.3, applied with group actions of the form given in Example 1.1(iii), we will deduce the following corollary.

Corollary 1.4. Suppose that $p>2$. Then (1.6) admits an unbounded sequence $\left(u_{n}\right)_{n}$ of nonradial sign-changing solutions with $I\left(u_{n}\right) \rightarrow \infty$ as $n \rightarrow \infty$.

This paper is organized as follows. In Section 2, we set up the variational framework for (1.6) and present some preliminary results. In Section 3, we give the proof Theorem 1.1. Section 4 is devoted to the proof of Theorem 1.2. Finally, in Section 5 we will complete the proofs of Theorem 1.3 and Corollary 1.4

Throughout the paper, we make use of the following notation. $L^{s}\left(\mathbb{R}^{2}\right), 1 \leq s \leq \infty$ denotes the usual Lebesgue space with the norm $|\cdot|_{s}$. For any $\rho>0$ and for any $z \in \mathbb{R}^{2}, B_{\rho}(z)$ denotes the ball of radius $\rho$ centered at $z$. As usual, $X^{\prime}$ denotes the dual space of $X$. Finally, $C, C_{1}, C_{2}, \cdots$ denote different positive constants whose exact value is inessential. 


\section{Preliminaries}

In the section, we recall the variational setting for (1.6) as elaborated by [6] and establish some useful preliminary results. Let $H^{1}\left(\mathbb{R}^{2}\right)$ be the usual Sobolev space endowed with the standard inner product

$$
\langle u, v\rangle=\int_{\mathbb{R}^{2}}(\nabla u \nabla v+u v) d x \quad \text { for } u, v \in H^{1}\left(\mathbb{R}^{2}\right),
$$

and the induced norm denoted by $\|u\|=\langle u, u\rangle^{1 / 2}$. We define the symmetric bilinear forms

$$
\begin{aligned}
& (u, v) \mapsto B_{1}(u, v)=\int_{\mathbb{R}^{2}} \int_{\mathbb{R}^{2}} \log (1+|x-y|) u(x) v(y) d x d y, \\
& (u, v) \mapsto B_{2}(u, v)=\int_{\mathbb{R}^{2}} \int_{\mathbb{R}^{2}} \log \left(1+\frac{1}{|x-y|}\right) u(x) v(y) d x d y, \\
& (u, v) \mapsto B_{0}(u, v)=B_{1}(u, v)-B_{2}(u, v)=\int_{\mathbb{R}^{2}} \int_{\mathbb{R}^{2}} \log (|x-y|) u(x) v(y) d x d y .
\end{aligned}
$$

Here, in each case, the definition is restricted to measurable functions $u, v: \mathbb{R}^{2} \rightarrow \mathbb{R}$ such that the corresponding double integral is well-defined in the Lebesgue sense. We remark that, since $0<\log (1+r)<r$ for $r>0$, from the Hardy-Littlewood-Sobolev inequality [14] we deduce that

$$
\left|B_{2}(u, v)\right| \leq \int_{\mathbb{R}^{2}} \int_{\mathbb{R}^{2}} \frac{1}{|x-y|}|u(x) v(y)| d x d y \leq C_{0}|u|_{\frac{4}{3}}|v|_{\frac{4}{3}} \quad \text { for } u, v \in L^{\frac{4}{3}}\left(\mathbb{R}^{2}\right)
$$

with a constant $C_{0}>0$. Then we define the functionals

$$
\begin{array}{lll}
V_{1}: H^{1}\left(\mathbb{R}^{2}\right) \rightarrow[0, \infty], & V_{1}(u)=B_{1}\left(u^{2}, u^{2}\right)=\int_{\mathbb{R}^{2}} \int_{\mathbb{R}^{2}} \log (1+|x-y|) u^{2}(x) u^{2}(y) d x d y, \\
V_{2}: L^{\frac{8}{3}}\left(\mathbb{R}^{2}\right) \rightarrow[0, \infty), & V_{2}(u)=B_{2}\left(u^{2}, u^{2}\right)=\int_{\mathbb{R}^{2}} \int_{\mathbb{R}^{2}} \log \left(1+\frac{1}{|x-y|}\right) u^{2}(x) u^{2}(y) d x d y, \\
V_{0}: H^{1}\left(\mathbb{R}^{2}\right) \rightarrow \mathbb{R} \cup\{\infty\}, & V_{0}(u)=B_{0}\left(u^{2}, u^{2}\right)=\int_{\mathbb{R}^{2}} \int_{\mathbb{R}^{2}} \log (|x-y|) u^{2}(x) u^{2}(y) d x d y .
\end{array}
$$

Note that, by (2.1) we have

$$
\left|V_{2}(u)\right| \leq C_{0}|u|_{\frac{8}{3}}^{4} \quad \text { for all } u \in L^{\frac{8}{3}}\left(\mathbb{R}^{2}\right)
$$

thus $V_{2}$ only takes finite values on $L^{\frac{8}{3}}\left(\mathbb{R}^{2}\right) \subset H^{1}\left(\mathbb{R}^{2}\right)$. Next, for any measurable function $u: \mathbb{R}^{2} \rightarrow \mathbb{R}$, we define

$$
|u|_{*}:=\left(\int_{\mathbb{R}^{2}} \log (1+|x|) u^{2} d x\right)^{1 / 2} \in[0, \infty] .
$$

Since

$$
\log (1+|x-y|) \leq \log (1+|x|+|y|) \leq \log (1+|x|)+\log (1+|y|) \quad \text { for } x, y \in \mathbb{R}^{2},
$$

we have the estimate

$$
\begin{aligned}
B_{1}(u v, w z) & \leq \int_{\mathbb{R}^{2}} \int_{\mathbb{R}^{2}}[\log (1+|x|)+\log (1+|y|)]|u(x) v(x)||w(y) z(y)| d x d y \\
& \leq|u|_{*}|v|_{*}|w|_{2}|z|_{2}+|u|_{2}|v|_{2}|w|_{*}|z|_{*} \quad \text { for } u, v, w, z \in L^{2}\left(\mathbb{R}^{2}\right)
\end{aligned}
$$

with the conventions $\infty \cdot 0=0$ and $\infty \cdot s=\infty$ for $s>0$. Some useful properties of $B_{1}$ are contained in the following lemmas from [6]. 
Lemma 2.1 ([6, Lemma 2.1]). Suppose that $\left\{u_{n}\right\}$ is a sequence in $L^{2}\left(\mathbb{R}^{2}\right)$ such that $u_{n} \rightarrow u \in$ $L^{2}\left(\mathbb{R}^{2}\right) \backslash\{0\}$ pointwise a.e. in $\mathbb{R}^{2}$. Moreover, let $\left\{v_{n}\right\}$ be a bounded sequence in $L^{2}\left(\mathbb{R}^{2}\right)$ such that

$$
\sup _{n \in \mathbb{N}} B_{1}\left(u_{n}^{2}, v_{n}^{2}\right)<\infty
$$

Then, there exist $n_{0} \in \mathbb{N}$ and $C>0$ such that $\left|v_{n}\right|_{*}<C$ for $n \geq n_{0}$. If moreover $B_{1}\left(u_{n}^{2}, v_{n}^{2}\right) \rightarrow 0$ and $\left|v_{n}\right|_{2} \rightarrow 0$ as $n \rightarrow \infty$, then $\left|v_{n}\right|_{*} \rightarrow 0$ as $n \rightarrow \infty$.

Lemma 2.2 ([6, Lemma 2.6]). Let $\left\{u_{n}\right\},\left\{v_{n}\right\}$ and $\left\{w_{n}\right\}$ be bounded sequences in $X$ such that $u_{n} \rightarrow u$ weakly in $X$. Then, for every $z \in X$, we have $B_{1}\left(v_{n} w_{n}, z\left(u_{n}-u\right)\right) \rightarrow 0$ as $n \rightarrow \infty$.

In the following, we fix $p>2$ and consider the functional $I: H^{1}\left(\mathbb{R}^{2}\right) \rightarrow \mathbb{R} \cup\{\infty\}$ defined by

$$
I(u)=\frac{1}{2}\|u\|^{2}+\frac{1}{4} V_{0}(u)-\frac{1}{p} \int_{\mathbb{R}^{2}}|u|^{p} d x .
$$

We also define the Hilbert space

$$
X:=\left\{u \in H^{1}\left(\mathbb{R}^{2}\right):|u|_{*}<\infty\right\}
$$

with the norm given by $\|u\|_{X}:=\sqrt{\|u\|^{2}+|u|_{*}^{2}}$. Note that, from (2.3) we see that the restriction of $I$ to $X$ (also denoted by $I$ in the sequel) only takes finite values in $\mathbb{R}$. We need the following facts proved in [6].

Lemma 2.3 ( [6, Lemma 2.2]).

(i) The space $X$ is compactly embedded in $L^{s}\left(\mathbb{R}^{2}\right)$ for all $s \in[2, \infty)$.

(ii) The functionals $V_{0}, V_{1}, V_{2}$ and $I$ are of class $C^{1}$ on $X$. Moreover,

$$
V_{i}^{\prime}(u) v=4 B_{i}\left(u^{2}, u v\right) \quad \text { for } u, v \in X \text { and } i=0,1,2 .
$$

(iii) $V_{2}$ is continuously differentiable on $L^{\frac{8}{3}}\left(\mathbb{R}^{2}\right)$.

(iv) $V_{1}$ is weakly lower semicontinuous on $H^{1}\left(\mathbb{R}^{2}\right)$.

(v) $I$ is weakly lower semicontinuous on $X$.

(vi) $I$ is lower semicontinuous on $H^{1}\left(\mathbb{R}^{2}\right)$.

Next, we provide a Pohozaev type identity for equation (1.6). The strategy of the proof is similar as e.g. in [4,9], but some differences occur due to the presence of the logarithmic Newtonian potential.

Lemma 2.4 (Pohozaev type identity). Suppose that $u \in X$ be a weak solution to (1.6). Then we have the following identity:

$$
P(u):=\int_{\mathbb{R}^{2}}|u|^{2} d x+\int_{\mathbb{R}^{2}} \int_{\mathbb{R}^{2}} \log (|x-y|) u^{2}(x) u^{2}(y) d x d y+\frac{1}{4}\left(\int_{\mathbb{R}^{2}} u^{2} d x\right)^{2}-\frac{2}{p} \int_{\mathbb{R}^{2}}|u|^{p} d x=0 .
$$

Proof. We first recall from Remark 1.1 that $u$ is of class $C^{2}$ with $u(x)=o\left(-e^{\alpha|x|}\right)$ as $|x| \rightarrow \infty$ for any $\alpha>0$, and that the function

$$
w: \mathbb{R}^{2} \rightarrow \mathbb{R}, \quad w(x)=\int_{\mathbb{R}^{2}} \log (|x-y|) u^{2}(y) d y
$$


is of class $C^{3}$. In this proof, we also consider the functions

$$
g(u)=|u|^{p-2} u-u \quad \text { and } \quad G(u)=\int_{0}^{u} g(s) d s=\frac{|u|^{p}}{p}-\frac{u^{2}}{2} \quad \in C^{2}(\mathbb{R})
$$

which also decay exponentially as $|x| \rightarrow \infty$. In the first part of the proof we use the device of Pohozaev [18, multiplying the equation by $x \cdot \nabla u$ and integrating by parts to get an integral identity on a ball $B_{R}(0)$. In the second part, we then show that the boundary term in the identity tends to zero as $R \rightarrow \infty$. So let $R>0$. Since, as noted e.g. in [25, p. 136], for any function $u \in C^{2}\left(\mathbb{R}^{2}\right)$ we have

$$
\Delta u(x \cdot \nabla u)=\operatorname{div}\left(\nabla u(x \cdot \nabla u)-x \frac{|\nabla u|^{2}}{2}\right) \quad \text { on } \mathbb{R}^{2},
$$

the divergence theorem gives

$$
\int_{B_{R}(0)}-\Delta u(x \cdot \nabla u) d x=-\frac{1}{R} \int_{\partial B_{R}(0)}|x \cdot \nabla u|^{2} d \sigma+\frac{R}{2} \int_{\partial B_{R}(0)}|\nabla u|^{2} d \sigma .
$$

Similarly, since $g(u)(x \cdot \nabla u)=\operatorname{div}(x G(u))-2 G(u)$ on $\mathbb{R}^{2}$, we have

$$
\int_{B_{R}(0)} g(u)(x \cdot \nabla u) d x=-2 \int_{B_{R}(0)} G(u) d x+R \int_{\partial B_{R}(0)} G(u) d \sigma
$$

Moreover, since $w u(x \cdot \nabla u)=\frac{1}{2}\left(\operatorname{div}\left[x w u^{2}\right]-u^{2}(x \cdot \nabla w)-2 w u^{2}\right)$, we have

$$
\int_{B_{R}(0)} w u(x \cdot \nabla u) d x=-\frac{1}{2} \int_{B_{R}(0)} u^{2}(x \cdot \nabla w) d x-\int_{B_{R}(0)} w u^{2} d x+\frac{R}{2} \int_{\partial B_{R}(0)} w u^{2} d \sigma .
$$

Thus, multiplying (1.6) by $x \cdot \nabla u$ and integrating on $B_{R}(0)$, we deduce from (2.5), (2.6) and (2.7) that

$$
\int_{B_{R}(0)}\left(\frac{u^{2}(x \cdot \nabla w)}{2}+w u^{2}-2 G(u)\right) d x=\int_{\partial B_{R}(0)}\left(-\frac{|x \cdot \nabla u|^{2}}{R}+R\left(\frac{|\nabla u|^{2}}{2}+\frac{w u^{2}}{2}-G(u)\right)\right) d \sigma .
$$

Next, following the idea in [4], we will show that the right hand side in (2.8) converges to zero for a suitable sequence $R_{n} \rightarrow \infty$, i.e.,

$$
R_{n} \int_{\partial B_{R_{n}}(0)}|f| d \sigma \rightarrow 0 \quad \text { for the function } x \mapsto f(x)=\frac{|\nabla u|^{2}}{2}+\frac{w u^{2}}{2}-G(u)-\frac{|x \cdot \nabla u|^{2}}{|x|^{2}} .
$$

For this it suffices to show that

$$
f \in L^{1}\left(\mathbb{R}^{2}\right)
$$

Indeed, if no sequence $\left(R_{n}\right)_{n}$ with $R_{n} \rightarrow \infty$ and (2.9) exists, it follows that

$$
\int_{\partial B_{R}(0)}|f| d \sigma \geq \frac{c}{R} \quad \text { for } R \geq R_{0} \text { with some constants } c, R_{0}>0 .
$$

This contradicts (2.10), as

$$
\int_{\mathbb{R}^{2}}|f| d x=\int_{0}^{\infty} d R \int_{\partial B_{R}(0)}|f| d \sigma \geq c \int_{R_{0}}^{\infty} \frac{1}{R} d R=\infty .
$$


To see (2.10), it suffices to recall from Remark 1.1 that $u$ and $\nabla u$ decay exponentially, whereas $w$ grows logarithmically as $|x| \rightarrow \infty$. Indeed, the exponential decay of $\nabla u$ follows by the decay of $u$ and standard elliptic regularity. Consequently, the function $f$ also decay exponentially as $|x| \rightarrow \infty$, which gives (2.10) and therefore (2.9). To conclude the proof, we note that, by the same arguments,

$$
w u^{2} \in L^{1}\left(\mathbb{R}^{2}\right) \text { and } G(u) \in L^{1}\left(\mathbb{R}^{2}\right) .
$$

Moreover a direct calculation gives

$$
x \cdot \nabla w(x)=\int_{\mathbb{R}^{2}} \frac{|x|^{2}-x \cdot y}{|x-y|^{2}} u^{2}(y) d y \quad \text { for } x \in \mathbb{R}^{2} .
$$

By the exponential decay of $u$, it then follows that $|x \cdot \nabla w(x)| \leq C|x|$ for $x \in \mathbb{R}^{2}$ with a constant $C>0$, and thus $u^{2}(x \cdot \nabla w) \in L^{1}\left(\mathbb{R}^{2}\right)$. Moreover,

$$
\begin{aligned}
\int_{\mathbb{R}^{2}} u^{2}(x \cdot \nabla w) d x=\int_{\mathbb{R}^{2}} \int_{\mathbb{R}^{2}} \frac{|x|^{2}-x \cdot y}{|x-y|^{2}} u^{2}(x) u^{2}(y) d y & =\frac{1}{2} \int_{\mathbb{R}^{2}} \int_{\mathbb{R}^{2}} \frac{|x|^{2}+|y|^{2}-2 x \cdot y}{|x-y|^{2}} u^{2}(x) u^{2}(y) d y \\
& =\frac{1}{2}\left(\int_{\mathbb{R}^{2}} u^{2} d x\right)^{2} .
\end{aligned}
$$

Consequently, with $P(u)$ as given in the statement of the lemma and $f$ given in (2.9) we have, by (2.8),

$$
\begin{aligned}
P(u)=\int_{\mathbb{R}^{2}}\left(\frac{u^{2}(x \cdot \nabla w)}{2}+w u^{2}-2 G(u)\right) d x & =\lim _{n \rightarrow \infty} \int_{B_{R_{n}}(0)}\left(\frac{u^{2}(x \cdot \nabla w)}{2} w u^{2}-2 G(u)\right) d x \\
& =\lim _{n \rightarrow \infty} R_{n} \int_{\partial B_{R_{n}}(0)}|f| d \sigma=0
\end{aligned}
$$

as claimed.

We close this section with some observations on the shape of the functional $I$.

Lemma 2.5. There exists $\rho>0$ such that

$$
m_{\beta}:=\inf \{I(u): u \in X,\|u\|=\beta\}>0 \quad \text { for } 0<\beta \leq \rho
$$

and

$$
n_{\beta}:=\inf \left\{I^{\prime}(u) u: u \in X,\|u\|=\beta\right\}>0 \quad \text { for } 0<\beta \leq \rho .
$$

Proof. For each $u \in X$, by (2.2) and Sobolev embeddings we have

$$
I(u) \geq \frac{\|u\|^{2}}{2}-\frac{V_{2}(u)}{4}-\frac{1}{p} \int_{\mathbb{R}^{2}}|u|^{p} d x \geq \frac{\|u\|^{2}}{2}-\frac{C_{0}}{4}|u|_{\frac{8}{3}}^{4}-\frac{1}{p}|u|_{p}^{p} \geq \frac{\|u\|^{2}}{2}\left(1-C_{1}\|u\|^{2}-C_{2}\|u\|^{p-2}\right),
$$

where $C_{0}, C_{1}, C_{2}>0$ are constants. This implies that (2.11) holds for $\rho>0$ sufficiently small. Since

$$
I^{\prime}(u)(u)=\|u\|^{2}+V_{0}(u)-|u|_{p}^{p} \geq\|u\|^{2}-V_{2}(u)-|u|_{p}^{p}
$$

for $u \in X$, a similar estimate shows that (2.12) holds for $\rho>0$ sufficiently small. 
Lemma 2.6. Let $u \in X \backslash\{0\}$, and let $u_{t} \in X$ be defined by $u_{t}(x):=t^{2} u(t x)$ for $x \in \mathbb{R}^{2}, t>0$. Then we have

$$
I\left(u_{t}\right) \rightarrow-\infty \quad \text { as } t \rightarrow \infty .
$$

In particular, the functional $I$ is not bounded from below.

Proof. Let $u \in X \backslash\{0\}$. Then we have

$$
\begin{aligned}
I\left(u_{t}\right)= & \frac{t^{4}}{2} \int_{\mathbb{R}^{2}}|\nabla u|^{2} d x+\frac{t^{2}}{2} \int_{\mathbb{R}^{2}} u^{2} d x+\frac{t^{4}}{4} \int_{\mathbb{R}^{2}} \int_{\mathbb{R}^{2}} \log (|x-y|) u^{2}(x) u^{2}(y) d x d y \\
& -\frac{t^{4} \log t}{4}\left(\int_{\mathbb{R}^{2}}|u|^{2} d x\right)^{2}-\frac{t^{2 p-2}}{p} \int_{\mathbb{R}^{2}}|u|^{p} d x .
\end{aligned}
$$

Consequently, $I\left(u_{t}\right) \rightarrow-\infty$ as $t \rightarrow \infty$, and the claim follows.

\section{Existence of mountain pass and ground state solutions to (1.6)}

In this section, we will prove Theorem 1.1. For this we will first prove the existence of critical points of $I$ at the mountain pass energy level $c_{m p}$ defined in (1.9). Within this step, we shall use the following general minimax principle from [13]. It is a somewhat stronger variant of [25, Theorem 2.8] which gives rise to Cerami sequences instead of Palais-Smale sequences.

Proposition 3.1 ([13, Proposition 2.8]). Let $X$ be a Banach space. Let $M_{0}$ be a closed subspace of the metric space $M$ and $\Gamma_{0} \subset C\left(M_{0}, X\right)$. Define

If $\varphi \in C^{1}(X, \mathbb{R})$ satisfies

$$
\Gamma:=\left\{\gamma \in C(M, X):\left.\gamma\right|_{M_{0}} \in \Gamma_{0}\right\} .
$$

$$
\infty>c:=\inf _{\gamma \in \Gamma} \sup _{u \in M} \varphi(\gamma(u))>a:=\sup _{\gamma_{0} \in \Gamma_{0}} \sup _{u \in M_{0}} \varphi\left(\gamma_{0}(u)\right),
$$

then, for every $\varepsilon \in\left(0, \frac{c-a}{2}\right), \delta>0$ and $\gamma \in \Gamma$ with $\sup _{u \in M} \varphi(\gamma(u)) \leq c+\varepsilon$ there exists $u \in X$ such that

(a) $c-2 \varepsilon \leq \varphi(u) \leq c+2 \varepsilon$,

(b) $\operatorname{dist}(u, \gamma(M)) \leq 2 \delta$,

(c) $\left(1+\|u\|_{X}\right)\left\|\varphi^{\prime}(u)\right\|_{X^{\prime}} \leq \frac{8 \varepsilon}{\delta}$.

We now consider the mountain pass value

$$
c_{m p}=\inf _{\gamma \in \Gamma} \max _{t \in[0,1]} I(\gamma(t)),
$$

where

$$
\Gamma=\{\gamma \in C([0,1], X) \mid \gamma(0)=0, I(\gamma(1))<0\} .
$$

By Lemmas 2.5 and 2.6, we find that

$$
0<m_{\rho} \leq c_{m p}<\infty
$$

which means that the functional $I$ has a mountain pass geometry. As the following lemma shows, we can now use Proposition 3.1 to prove the existence of a Cerami sequence $\left\{u_{n}\right\} \subset X$ at the energy level $c_{m p}$ with a key additional property. For Palais-Smale sequences in related variational settings, this idea goes back to [12] and has also been used in [11,17]. 
Lemma 3.2. Let $p>2$. Then there exists a sequence $\left\{u_{n}\right\}$ in $X$ such that, as $n \rightarrow \infty$,

$$
I\left(u_{n}\right) \rightarrow c_{m p}, \quad\left\|I^{\prime}\left(u_{n}\right)\right\|_{X^{\prime}}\left(1+\left\|u_{n}\right\|_{X}\right) \rightarrow 0 \quad \text { and } \quad J\left(u_{n}\right) \rightarrow 0
$$

where $J: X \rightarrow \mathbb{R}$ is defined by (1.11).

Proof. Following the strategy of [11,12,17], we consider the Banach space

$$
\tilde{X}:=\mathbb{R} \times X
$$

equipped with the standard product norm $\|(s, v)\|_{\tilde{X}}:=\left(|s|^{2}+\|v\|_{X}\right)^{1 / 2}$ for $s \in \mathbb{R}, v \in X$. Moreover, we define the continuous map

$$
\rho: \tilde{X} \rightarrow X, \quad \rho(s, v)[x]:=e^{2 s} v\left(e^{s} x\right) \quad \text { for } s \in \mathbb{R}, v \in X \text { and } x \in \mathbb{R}^{2} .
$$

We also consider the functional

$$
\varphi:=I \circ \rho: \tilde{X} \rightarrow \mathbb{R}
$$

A short computation yields that

$$
\begin{aligned}
\varphi(s, v)=I(\rho(s, v))= & \frac{e^{4 s}}{2} \int_{\mathbb{R}^{2}}|\nabla v|^{2} d x+\frac{e^{2 s}}{2} \int_{\mathbb{R}^{2}} v^{2} d x+\frac{e^{4 s}}{4} \int_{\mathbb{R}^{2}} \int_{\mathbb{R}^{2}} \log (|x-y|) v^{2}(x) v^{2}(y) d x d y \\
& -\frac{s e^{4 s}}{4}\left(\int_{\mathbb{R}^{2}}|v|^{2} d x\right)^{2}-\frac{e^{2 s(p-1)}}{p} \int_{\mathbb{R}^{2}}|v|^{p} d x \quad \text { for } s \in \mathbb{R} \text { and } v \in X . \quad(3.2)
\end{aligned}
$$

This readily implies that $\varphi$ is of class $C^{1}$ on $X$ with

$$
\begin{aligned}
\partial_{s} \varphi(s, v)= & 2 e^{4 s} \int_{\mathbb{R}^{2}}|\nabla v|^{2} d x+e^{2 s} \int_{\mathbb{R}^{2}} v^{2} d x+e^{4 s} \int_{\mathbb{R}^{2}} \int_{\mathbb{R}^{2}} \log (|x-y|) v^{2}(x) v^{2}(y) d x d y \\
& -\left(s e^{4 s}+\frac{e^{4 s}}{4}\right)\left(\int_{\mathbb{R}^{2}}|v|^{2} d x\right)^{2}-\frac{2(p-1)}{p} e^{2 s(p-1)} \int_{\mathbb{R}^{2}}|v|^{p} d x \\
= & 2 \int_{\mathbb{R}^{2}}|\nabla \rho(s, v)|^{2} d x+\int_{\mathbb{R}^{2}} \rho(s, v)^{2} d x+\int_{\mathbb{R}^{2}} \int_{\mathbb{R}^{2}} \log (|x-y|) \rho(s, v)^{2}(x) \rho(s, v)^{2}(y) d x d y \\
& -\frac{1}{4}\left(\int_{\mathbb{R}^{2}}|\rho(s, v)|^{2} d x\right)^{2}-\frac{2(p-1)}{p} \int_{\mathbb{R}^{2}}|\rho(s, v)|^{p} d x \\
= & J(\rho(s, v)) \quad \text { for } s \in \mathbb{R} \text { and } v \in X,
\end{aligned}
$$

where $J$ defined in (1.11). Moreover, since the map $v \mapsto \rho(s, v)$ is linear for fixed $s \in \mathbb{R}$, we have

$$
\partial_{v} \varphi(s, v) w=I^{\prime}(\rho(s, v)) \rho(s, w) \quad \text { for } s \in \mathbb{R} \text { and } v, w \in X .
$$

Next, we define the minimax value

$$
c_{*}=\inf _{\tilde{\gamma} \in \tilde{\Gamma}} \max _{t \in[0,1]} \varphi(\tilde{\gamma}(t))
$$

where

$$
\tilde{\Gamma}:=\{\tilde{\gamma} \in C([0,1], \tilde{X}) \mid \tilde{\gamma}(0)=(0,0), \varphi(\tilde{\gamma}(1))<0\}
$$


Since $\Gamma=\{\rho \circ \tilde{\gamma}: \tilde{\gamma} \in \tilde{\Gamma}\}$, the minimax values of $I$ and $\varphi$ coincide, i.e., $c_{m p}=c_{*}$. We will now apply Proposition 3.1 to the functional $\varphi, M=[0,1], M_{0}=\{0,1\}$ and $\tilde{X}, \tilde{\Gamma}$ in place of $X, \Gamma$. More precisely, for fixed $n \in \mathbb{N}$ we use the definition of $c_{m p}$ and choose $\gamma_{n} \in \Gamma$ with

$$
\max _{t \in[0,1]} I\left(\gamma_{n}(t)\right) \leq c_{m p}+\frac{1}{n^{2}}
$$

We then define $\tilde{\gamma}_{n} \in \tilde{\Gamma}$ by $\tilde{\gamma}_{n}(t)=\left(0, \gamma_{n}(t)\right)$, and we note that

$$
\max _{t \in[0,1]} \varphi\left(\tilde{\gamma}_{n}(t)\right)=\max _{t \in[0,1]} I\left(\gamma_{n}(t)\right) \leq c_{m p}+\frac{1}{n^{2}}
$$

An application of Proposition 3.1 with $\tilde{\gamma}_{n}$ in place of $\gamma$ and $\varepsilon=\frac{1}{n^{2}}, \delta=\frac{1}{n}$ now yields the existence of $\left(s_{n}, v_{n}\right) \in \tilde{X}$ such that, as $n \rightarrow \infty$,

$$
\begin{aligned}
& \varphi\left(s_{n}, v_{n}\right) \rightarrow c_{m p}, \\
& \left\|\varphi^{\prime}\left(s_{n}, v_{n}\right)\right\|_{\tilde{X}^{\prime}}\left(1+\left\|\left(s_{n}, v_{n}\right)\right\|_{\tilde{X}}\right) \rightarrow 0, \\
& \operatorname{dist}\left(\left(s_{n}, v_{n}\right),\{0\} \times \gamma_{n}([0,1])\right) \rightarrow 0,
\end{aligned}
$$

whereas (3.7) obviously implies that

$$
s_{n} \rightarrow 0
$$

Since

$$
\varphi^{\prime}\left(s_{n}, v_{n}\right)(h, w)=I^{\prime}\left(\rho\left(s_{n}, v_{n}\right)\right) \rho\left(s_{n}, w\right)+J\left(\rho\left(s_{n}, v_{n}\right)\right) h \quad \text { for }(h, w) \in \tilde{X}
$$

by (3.3) and (3.4), we may take $h=1$ and $w=0$ in (3.9) to obtain

$$
J\left(\rho\left(s_{n}, v_{n}\right)\right) \rightarrow 0 \quad \text { as } n \rightarrow \infty .
$$

For $u_{n}:=\rho\left(s_{n}, v_{n}\right)$, it then follows from (3.5) and (3.10) that

$$
I\left(u_{n}\right) \rightarrow c_{m p} \quad \text { and } \quad J\left(u_{n}\right) \rightarrow 0 \quad \text { as } n \rightarrow \infty .
$$

Finally, for given $v \in X$ we consider $w_{n}=e^{-2 s_{n}} v\left(e^{-s_{n}} \cdot\right) \in X$ and deduce from (3.6) and (3.9) with $h=0$ that

$$
\left(1+\left\|u_{n}\right\|_{X}\right)\left|I^{\prime}\left(u_{n}\right) v\right|=\left(1+\left\|u_{n}\right\|_{X}\right)\left|I^{\prime}\left(u_{n}\right) \rho\left(s_{n}, w_{n}\right)\right|=o(1)\left\|w_{n}\right\|_{X} \quad \text { as } n \rightarrow \infty,
$$

whereas by (3.8) we have

$$
\begin{aligned}
\left\|w_{n}\right\|_{X}^{2} & =\left\|w_{n}\right\|^{2}+\left|w_{n}\right|_{*}^{2} \\
& =e^{-4 s_{n}} \int_{\mathbb{R}^{2}}|\nabla v|^{2} d x+e^{-2 s_{n}} \int_{\mathbb{R}^{2}}\left(1+\log \left(1+e^{2 s_{n}}|x|\right)\right) v^{2} d x \\
& =[1+o(1)] \int_{\mathbb{R}^{2}}|\nabla v|^{2} d x+[1+o(1)] \int_{\mathbb{R}^{2}}(1+\log (1+|x|)) v^{2} d x \\
& =(1+o(1))\|v\|_{X}^{2} \quad \text { as } n \rightarrow \infty
\end{aligned}
$$

with $o(1) \rightarrow 0$ uniformly in $v \in X$. Combining the latter two estimates, we get that

$$
\left(1+\left\|u_{n}\right\|_{X}\right)\left\|I^{\prime}\left(u_{n}\right)\right\|_{X^{\prime}} \rightarrow 0 \quad \text { as } n \rightarrow \infty .
$$

The proof is thus finished. 
In the following key proposition, we shall show, in particular, that any sequence $\left(u_{n}\right)_{n}$ satisfying (3.1) is bounded in $H^{1}\left(\mathbb{R}^{2}\right)$.

Proposition 3.3. Let $p>2$, and $\left\{u_{n}\right\}$ be a sequence in $X$ such that

$$
c:=\sup _{n \in \mathbb{N}} I\left(u_{n}\right)<\infty \quad \text { and } \quad\left\|I^{\prime}\left(u_{n}\right)\right\|_{X^{\prime}}\left(1+\left\|u_{n}\right\|_{X}\right) \rightarrow 0, \quad J\left(u_{n}\right) \rightarrow 0 \quad \text { as } n \rightarrow \infty .
$$

Then $\left(u_{n}\right)_{n}$ is bounded in $H^{1}\left(\mathbb{R}^{2}\right)$.

Proof. In the following, $C_{1}, C_{2}, \cdots$ denote positive constants independent of $n \in \mathbb{N}$. We first observe from (3.11) that

$$
c+o(1) \geq I\left(u_{n}\right)-\frac{1}{4} J\left(u_{n}\right)=\frac{1}{4}\left|u_{n}\right|_{2}^{2}+\frac{1}{16}\left|u_{n}\right|_{2}^{4}+\frac{p-3}{2 p}\left|u_{n}\right|_{p}^{p} .
$$

We may then distinguish the following two cases:

Case 1: $p>3$. In this case, (3.12) implies that $\left(u_{n}\right)_{n}$ is bounded in $L^{2}\left(\mathbb{R}^{2}\right)$ and in $L^{p}\left(\mathbb{R}^{2}\right)$. Therefore, by (2.2) we have

$$
V_{2}\left(u_{n}\right) \leq C_{0}\left|u_{n}\right|_{\frac{8}{3}}^{4} \leq C_{0}\left|u_{n}\right|_{2}^{4\left(1-\theta_{0}\right)}\left|u_{n}\right|_{p}^{4 \theta_{0}} \leq C_{1}
$$

where $\theta_{0}=\frac{p}{4(p-2)}$. Consequently, we may use (3.11) again to estimate

$$
2\left\|u_{n}\right\|^{2}+V_{1}\left(u_{n}\right)=4 I\left(u_{n}\right)+V_{2}\left(u_{n}\right)+\frac{4}{p}\left|u_{n}\right|_{p}^{p} \leq 4 c+C_{1}+\frac{4}{p}\left|u_{n}\right|_{p}^{p} \leq C_{2} .
$$

This implies that $\left\{u_{n}\right\}$ is bounded in $H^{1}\left(\mathbb{R}^{2}\right)$.

Case 2: $2<p \leq 3$. We first claim that

$$
\left|\nabla u_{n}\right|_{2} \leq C_{3} \quad \text { for } n \in \mathbb{N} .
$$

Suppose by contradiction that this is false. Then, after passing to a subsequence, we have

$$
\left|\nabla u_{n}\right|_{2} \rightarrow \infty \quad \text { as } n \rightarrow \infty
$$

Let $t_{n}:=\left|\nabla u_{n}\right|_{2}^{-1 / 2}$ for $n \in \mathbb{N}$, so that $t_{n} \rightarrow 0$ as $n \rightarrow \infty$. For $n \in \mathbb{N}$ we define the rescaled functions $v_{n} \in X$ by $v_{n}(x):=t_{n}^{2} u_{n}\left(t_{n} x\right)$ for $n \in \mathbb{N}$, so that

$$
\left|\nabla v_{n}\right|_{2}=1 \quad \text { and } \quad\left|v_{n}\right|_{q}^{q}=t_{n}^{2 q-2}\left|u_{n}\right|_{q}^{q} \quad \text { for all } n \in \mathbb{N}, 1 \leq q<\infty .
$$

By the Gagliardo-Nirenberg inequality,

$$
\left|v_{n}\right|_{p}^{p} \leq C_{4}\left|v_{n}\right|_{2}^{2}\left|\nabla v_{n}\right|_{2}^{p-2}=C_{4}\left|v_{n}\right|_{2}^{2} \quad \text { for } n \in \mathbb{N} .
$$

Multiplying (3.12) by $t_{n}^{4}$, we deduce, from (3.15) and (3.16),

$$
\begin{aligned}
c t_{n}^{4}+o\left(t_{n}^{4}\right)=\frac{t_{n}^{4}}{4}\left|u_{n}\right|_{2}^{2}+\frac{t_{n}^{4}}{16}\left|u_{n}\right|_{2}^{4}-\frac{3-p}{2 p} t_{n}^{4}\left|u_{n}\right|_{p}^{p} & =\frac{t_{n}^{2}}{4}\left|v_{n}\right|_{2}^{2}+\frac{1}{16}\left|v_{n}\right|_{2}^{4}-\frac{3-p}{2 p} t_{n}^{6-2 p}\left|v_{n}\right|_{p}^{p} \\
& \geq \frac{t_{n}^{2}}{4}\left|v_{n}\right|_{2}^{2}+\frac{1}{16}\left|v_{n}\right|_{2}^{4}-\frac{3-p}{2 p} C_{4} t_{n}^{6-2 p}\left|v_{n}\right|_{2}^{2} .
\end{aligned}
$$


Consequently,

$$
\left|v_{n}\right|_{2}= \begin{cases}o\left(t_{n}^{1 / 2}\right) & \text { if } p=3 \\ o\left(t_{n}^{(3-p) / 2}\right) & \text { if } 2<p<3 .\end{cases}
$$

Moreover, by assumption we also have that

$$
o(1)=t_{n}^{4} J\left(u_{n}\right)=t_{n}^{4}\left(2\left|\nabla u_{n}\right|_{2}^{2}+\left|u_{n}\right|_{2}^{2}+V_{0}\left(u_{n}\right)-\frac{\left|u_{n}\right|_{2}^{4}}{4}-\frac{2 p-2}{p}\left|u_{n}\right|_{p}^{p}\right) .
$$

Combining this with (3.15), (3.17) and the fact that

$$
V_{0}\left(u_{n}\right)=t_{n}^{4} \int_{\mathbb{R}^{2}} \int_{\mathbb{R}^{2}} \log \left(\left|t_{n} x-t_{n} y\right|\right) u_{n}^{2}\left(t_{n} x\right) u_{n}^{2}\left(t_{n} y\right) d x d y=t_{n}^{-4}\left(V_{0}\left(v_{n}\right)+\left|v_{n}\right|_{2}^{4} \log t_{n}\right)
$$

we infer that

$$
\begin{aligned}
o(1) & =2+t_{n}^{2}\left|v_{n}\right|_{2}^{2}+V_{0}\left(v_{n}\right)+\left|v_{n}\right|_{2}^{4} \log t_{n}-\frac{1}{4}\left|v_{n}\right|_{2}^{4}-\frac{2 p-2}{p} t_{n}^{6-2 p}\left|v_{n}\right|_{p}^{p} \\
& =2+V_{0}\left(v_{n}\right)+o(1) .
\end{aligned}
$$

Since $V_{0}=V_{1}-V_{2}$, it now follows from (2.2), (3.17), (3.18) and the Gagliardo-Nirenberg inequality that

$$
2+V_{1}\left(v_{n}\right)=V_{2}\left(v_{n}\right)+o(1) \leq C_{0}\left|v_{n}\right|_{\frac{8}{3}}^{4}+o(1) \leq C_{5}\left|v_{n}\right|_{2}^{3}+o(1)=o(1) .
$$

Since $V_{1}$ is nonnegative, this is a contradiction. We thus conclude that (3.13) holds, and using (3.11) again we may then deduce that

$$
\frac{1}{4}\left|u_{n}\right|_{2}^{4}+\frac{p-2}{p}\left|u_{n}\right|_{p}^{p}=\left|\nabla u_{n}\right|_{2}^{2}+I^{\prime}\left(u_{n}\right) u_{n}-J\left(u_{n}\right) \leq C_{3}+o(1) .
$$

Consequently, $\left(u_{n}\right)_{n}$ is bounded in $L^{2}\left(\mathbb{R}^{2}\right)$, and together with (3.13) this shows that $\left(u_{n}\right)_{n}$ is bounded in $H^{1}\left(\mathbb{R}^{2}\right)$, as claimed.

We now define, for a function $u \in \mathbb{R}^{2} \rightarrow \mathbb{R}$ and $z \in \mathbb{R}^{2}$, the translated function

$$
z * u: \mathbb{R}^{2} \rightarrow \mathbb{R}, \quad(z * u)(x)=u(x-z) \quad \text { for } x \in \mathbb{R}^{2}
$$

We then may derive the following compactness property (modulo translation) for the class of Cerami sequence satisfying (3.11). It is a variant of [6, Proposition 3.1] based on Proposition 3.3.

Proposition 3.4. Let $p>2$, and let $\left(u_{n}\right)_{n}$ be a sequence in $X$ that satisfies (3.11). Then, after passing to a subsequence, one of the following occurs:

(I) $\left\|u_{n}\right\| \rightarrow 0$ and $I\left(u_{n}\right) \rightarrow 0$ as $n \rightarrow \infty$.

(II) There exist points $y_{n} \in \mathbb{R}^{2}, n \in \mathbb{N}$ such that

$$
y_{n} * u_{n} \rightarrow u \quad \text { strongly in } X \text { as } n \rightarrow \infty
$$

for some nonzero critical point $u \in X$ of $I$. 
Proof. We first note that $\left(u_{n}\right)_{n}$ is bounded in $H^{1}\left(\mathbb{R}^{2}\right)$ by Proposition 3.3. Suppose that (I) does not hold for any subsequence of $\left(u_{n}\right)_{n}$. We then claim that

$$
\liminf _{n \rightarrow \infty} \sup _{y \in \mathbb{R}^{2}} \int_{B_{2}(y)} u_{n}^{2}(x) d x>0 .
$$

Assuming the contrary that (3.19) does not occur. By Lions' vanishing lemma (see e.g. [15, 25]), after passing to a subsequence, it follows that

$$
u_{n} \rightarrow 0 \quad \text { in } L^{s}\left(\mathbb{R}^{2}\right) \quad \text { for all } s>2 \text {. }
$$

Therefore, by (2.2) and (3.11) we have

$$
\|u\|_{n}^{2}+V_{1}\left(u_{n}\right)=I^{\prime}\left(u_{n}\right) u_{n}+V_{2}\left(u_{n}\right)+\left|u_{n}\right|_{p}^{p} \rightarrow 0 \quad \text { as } n \rightarrow \infty .
$$

Hence, we obtain $\left\|u_{n}\right\| \rightarrow 0$ and $V_{1}\left(u_{n}\right) \rightarrow 0$, and thus

$$
I\left(u_{n}\right)=\frac{1}{2}\left\|u_{n}\right\|^{2}+\frac{1}{4}\left(V_{1}\left(u_{n}\right)-V_{2}\left(u_{n}\right)\right)-\frac{1}{p}\left|u_{n}\right|_{p}^{p} \rightarrow 0 \quad \text { as } n \rightarrow \infty .
$$

This contradicts our assumption that (I) does not hold for any such subsequence. So, (3.19) holds. Going if necessary to a subsequence, there exists a sequence $\left\{y_{n}\right\} \subset \mathbb{R}^{2}$ such that, the sequence of the functions

$$
\tilde{u}_{n}:=y_{n} * u_{n} \in X \quad \text { with } n \in \mathbb{N} \text {, }
$$

converges weakly in $H^{1}\left(\mathbb{R}^{2}\right)$ to some function $u \in H^{1}\left(\mathbb{R}^{2}\right) \backslash\{0\}$. Consequently, we may assume that $\tilde{u}_{n}(x) \rightarrow u(x)$ a.e. in $\mathbb{R}^{2}$. Moreover, using (3.11) again, we deduce that

$$
B_{1}\left(\tilde{u}_{n}^{2}, \tilde{u}_{n}^{2}\right)=V_{1}\left(\tilde{u}_{n}\right)=V_{1}\left(u_{n}\right)=o(1)+V_{2}\left(u_{n}\right)+\left|u_{n}\right|_{p}^{p}-\left\|u_{n}\right\|^{2},
$$

and the RHS of this inequality remains bounded in $n$. Thus, Lemma 2.1 implies that $\left|\tilde{u}_{n}\right|_{*}$ remains bounded in $n$, so that the sequence $\left\{\tilde{u}_{n}\right\}$ is bounded in $X$. Then, passing to a subsequence again if necessary, we may assume that $\tilde{u}_{n} \rightarrow u$ weakly in $X$, so that $u \in X$. It then follows from Lemma 2.3(i) that $\tilde{u}_{n} \rightarrow u$ strongly in $L^{s}\left(\mathbb{R}^{2}\right)$ for $s \geq 2$. Next, we claim that

$$
I^{\prime}\left(\tilde{u}_{n}\right)\left(\tilde{u}_{n}-u\right) \rightarrow 0 \quad \text { as } n \rightarrow \infty .
$$

Indeed, we have

$$
\left|I^{\prime}\left(\tilde{u}_{n}\right)\left(\tilde{u}_{n}-u\right)\right|=\left|I^{\prime}\left(u_{n}\right)\left(u_{n}-\left(-y_{n}\right) * u\right)\right| \leq\left\|I^{\prime}\left(u_{n}\right)\right\|_{X^{\prime}}\left(\left\|u_{n}\right\|_{X}+\left\|\left(-y_{n}\right) * u\right\|_{X}\right)
$$

for every $n$. Moreover, we can easily see that

$$
\left|u_{n}\right|_{*}^{2}=\int_{\mathbb{R}^{2}} \log \left(1+\left|x-y_{n}\right|\right) \tilde{u}_{n}^{2}(x) d x \geq C_{1} \log \left(1+\left|y_{n}\right|\right) \quad \text { for all } n
$$

with a constant $C_{1}>0$ and

$$
\left|\left(-y_{n}\right) * u\right|_{*}^{2}=\int_{\mathbb{R}^{2}} \log \left(1+\left|x-y_{n}\right|\right) u^{2}(x) d x \leq C_{2} \log \left(1+\left|y_{n}\right|\right) \quad \text { for all } n
$$


with a constant $C_{2}>0$. Combining these two inequalities with (3.19), we then find a constant $C_{3}>0$ such that, after passing to a subsequence,

$$
\left\|\left(-y_{n}\right) * u\right\|_{X}^{2}=\|u\|^{2}+\left|\left(-y_{n}\right) * u\right|_{*}^{2} \leq C_{3}\left(\left\|u_{n}\right\|^{2}+\left|u_{n}\right|_{*}^{2}\right)=C_{3}\left\|u_{n}\right\|_{X}^{2}
$$

for all $n \in \mathbb{N}$. Hence (3.21) implies that

$$
\left|I^{\prime}\left(\tilde{u}_{n}\right)\left(\tilde{u}_{n}-u\right)\right| \leq\left(1+\sqrt{C_{3}}\right)\left\|I^{\prime}\left(u_{n}\right)\right\|_{X^{\prime}}\left\|u_{n}\right\|_{X} \rightarrow 0 \quad \text { as } n \rightarrow \infty,
$$

as claimed in (3.20). Using (3.20), we conclude that

$$
\begin{aligned}
o(1) & =I^{\prime}\left(\tilde{u}_{n}\right)\left(\tilde{u}_{n}-u\right) \\
& =o(1)+\left\|\tilde{u}_{n}\right\|^{2}-\|u\|^{2}+\frac{1}{4} V_{0}^{\prime}\left(\tilde{u}_{n}\right)\left(\tilde{u}_{n}-u\right)-\int_{\mathbb{R}^{2}}\left|\tilde{u}_{n}\right|^{p-2} \tilde{u}_{n}\left(\tilde{u}_{n}-u\right) d x \\
& =o(1)+\left\|\tilde{u}_{n}\right\|^{2}-\|u\|^{2}+\frac{1}{4}\left[V_{1}^{\prime}\left(\tilde{u}_{n}\right)\left(\tilde{u}_{n}-u\right)-V_{2}^{\prime}\left(\tilde{u}_{n}\right)\left(\tilde{u}_{n}-u\right)\right],
\end{aligned}
$$

where

$$
\left|\frac{1}{4} V_{2}^{\prime}\left(\tilde{u}_{n}\right)\left(\tilde{u}_{n}-u\right)\right|=\left|B_{2}\left(\tilde{u}_{n}^{2}, \tilde{u}_{n}\left(\tilde{u}_{n}-u\right)\right)\right| \leq\left|\tilde{u}_{n}\right|_{\frac{8}{3}}^{3}\left|\tilde{u}_{n}-u\right|_{\frac{8}{3}} \rightarrow 0 \quad \text { as } n \rightarrow \infty
$$

and

$$
\frac{1}{4} V_{1}^{\prime}\left(\tilde{u}_{n}\right)\left(\tilde{u}_{n}-u\right)=B_{1}\left(\tilde{u}_{n}^{2}, \tilde{u}_{n}\left(\tilde{u}_{n}-u\right)\right)=B_{1}\left(\tilde{u}_{n}^{2},\left(\tilde{u}_{n}-u\right)^{2}\right)+B_{1}\left(\tilde{u}_{n}^{2}, u\left(\tilde{u}_{n}-u\right)\right)
$$

with

$$
B_{1}\left(\tilde{u}_{n}^{2}, u\left(\tilde{u}_{n}-u\right)\right) \rightarrow 0 \quad \text { as } n \rightarrow \infty
$$

by Lemma 2.2. Combining these estimates, we infer that

$$
o(1)=\left\|\tilde{u}_{n}\right\|^{2}-\|u\|^{2}+B_{1}\left(\tilde{u}_{n}^{2},\left(\tilde{u}_{n}-u\right)^{2}\right)+o(1) \geq\left\|\tilde{u}_{n}\right\|^{2}-\|u\|^{2}+o(1),
$$

which implies that $\left\|\tilde{u}_{n}\right\| \rightarrow\|u\|$ and $B_{1}\left(\tilde{u}_{n}^{2},\left(\tilde{u}_{n}-u\right)^{2}\right) \rightarrow 0$ as $n \rightarrow \infty$. Therefore, $\left\|\tilde{u}_{n}-u\right\| \rightarrow 0$ as $n \rightarrow \infty$. Moreover, by Lemma 2.1 we have $\left|\tilde{u}_{n}-u\right|_{*} \rightarrow 0$. We thus deduce that $\left\|\tilde{u}_{n}-u\right\|_{X} \rightarrow 0$ as $n \rightarrow \infty$, as claimed.

Finally, we need to show that $I^{\prime}(u)=0$. Let $v \in X$. Then, by the same argument which leads to (3.22), we obtain

$$
\left\|\left(-y_{n}\right) * v\right\|_{X} \leq C_{4}\left\|u_{n}\right\|_{X} \quad \text { for all } n
$$

with a constant $C_{4}>0$. So, from (3.11) we deduce that

$$
\begin{aligned}
\left|I^{\prime}(u) v\right| & =\lim _{n \rightarrow \infty}\left|I^{\prime}\left(\tilde{u}_{n}\right) v\right|=\lim _{n \rightarrow \infty}\left|I^{\prime}\left(u_{n}\right)\left[\left(-y_{n}\right) * v\right]\right| \\
& \leq \lim _{n \rightarrow \infty}\left\|I^{\prime}\left(u_{n}\right)\right\|_{X^{\prime}}\left\|\left(-y_{n}\right) * v\right\|_{X} \leq C_{4} \lim _{n \rightarrow \infty}\left\|I^{\prime}\left(u_{n}\right)\right\|_{X^{\prime}}\left\|u_{n}\right\|_{X}=0 .
\end{aligned}
$$

This completes the proof.

Proof of Theorem 1.1. By Lemma 3.2 and Proposition 3.4, there exists a critical point $u \in X \backslash\{0\}$ of $I$ with $I(u)=c_{m p}$, which already completes the proof of Theorem 1.1(i). In particular, the set

$$
\mathcal{K}=\left\{u \in X \backslash\{0\}: I^{\prime}(u)=0\right\}
$$


is nonempty. Let $\left(u_{n}\right)_{n} \subset \mathcal{K}$ be a sequence such that

$$
I\left(u_{n}\right) \rightarrow c_{g}=\inf _{u \in \mathcal{K}} I(u) \in\left[-\infty, c_{m p}\right] .
$$

By definition of $\mathcal{K}$ and Lemma 2.4, the sequence $\left(u_{n}\right)_{n}$ satisfies (3.11). Moreover, by (2.12) we have

$$
\liminf _{n \rightarrow \infty}\left\|u_{n}\right\| \geq \rho>0
$$

Consequently, by Proposition 3.4 there exist, after passing to a subsequence, points $x_{n} \in \mathbb{R}^{2}, n \in \mathbb{N}$ and a nonzero critical point $u \in X$ of $I$ such that

$$
x_{n} * u_{n} \rightarrow u \text { strongly in } X \text { as } n \rightarrow \infty
$$

So $u \in \mathcal{K}$ and

$$
I(u)=\lim _{n \rightarrow \infty} I\left(x_{n} * u_{n}\right)=\lim _{n \rightarrow \infty} I\left(u_{n}\right)=c_{g} .
$$

In particular we have $c_{g}>-\infty$, and $u$ has the properties asserted in Theorem 1.1(ii).

\section{Proof of Theorem 1.2}

In this section, we will give the proof of Theorem 1.2, For this we need to elaborate the saddle point structure of the functional $I$ in the case $p \geq 3$. We need the following lemma.

Lemma 4.1. Let $C_{i} \in \mathbb{R}, C_{i}>0$ for $i=1,3,4$, and let $C_{2} \in \mathbb{R}$. If $p \geq 3$, then the function

$$
f:(0, \infty) \rightarrow \mathbb{R}, \quad f(t)=C_{1} t^{2}+C_{2} t^{4}-C_{3} t^{4} \log t-C_{4} t^{2 p-2}
$$

has a unique positive critical point $t_{0}$ such that $f^{\prime}(t)>0$ for $t<t_{0}$ and $f^{\prime}(t)>0$ for $t>0$.

Proof. The proof is elementary, so we omit it.

Similarly as in [20], we now consider the functional $J$ defined in (1.11) and set $\mathcal{M}$ defined in (1.12), i.e.,

$$
\mathcal{M}=\{u \in X \backslash\{0\}: J(u)=0\}
$$

We then have

$$
J(u)=2 I^{\prime}(u) u-P(u),
$$

where $P(u)$ is given in Lemma 2.4. As already noted in the introduction, it follows from Lemma 2.4 that every critical point of $I$ is contained in $\mathcal{M}$ : Indeed this is true for arbitrary $p>2$.

In the following, for $u \in X$ and $t>0$, we set $Q(t, u):=u_{t} \in X \backslash\{0\}$, i.e.,

$$
Q(t, u)(x):=u_{t}(x)=t^{2} u(t x) \quad \text { for } x \in \mathbb{R}^{2} .
$$

Lemma 4.2. Let $p \geq 3$.

(i) For any $u \in X \backslash\{0\}$, there exists a unique $t_{u}>0$ such that $Q\left(t_{u}, u\right) \in \mathcal{M}$.

(ii) For any $u \in X \backslash\{0\}, t_{u}$ is the unique maximum point of the function $(0, \infty) \rightarrow \mathbb{R}, t \mapsto$ $I(Q(t, u))$. 
(iii) The map $X \backslash\{0\} \rightarrow(0, \infty), u \mapsto t_{u}$ is continuous.

(iv) Every $u \in \mathcal{M}$ with $I(u)=c_{\mathcal{M}}$ is a critical point of $I$ which does not change sign on $\mathbb{R}^{2}$.

Proof. For $u \in X \backslash\{0\}$, consider the function $h_{u}:(0, \infty) \rightarrow \mathbb{R}, h_{u}(t):=I(Q(t, u))$. As in (3.2), we see that

$$
\begin{aligned}
h_{u}(t)= & \frac{t^{4}}{2} \int_{\mathbb{R}^{2}}|\nabla u|^{2} d x+\frac{t^{2}}{2} \int_{\mathbb{R}^{2}} u^{2} d x+\frac{t^{4}}{4} \int_{\mathbb{R}^{2}} \int_{\mathbb{R}^{2}} \log (|x-y|) u^{2}(x) u^{2}(y) d x d y \\
& -\frac{t^{4} \log t}{4}\left(\int_{\mathbb{R}^{2}}|u|^{2} d x\right)^{2}-\frac{t^{2 p-2}}{p} \int_{\mathbb{R}^{2}}|u|^{p} d x .
\end{aligned}
$$

By Lemma 4.1, $h$ has a unique critical point $t_{u}>0$ such that

$$
h_{u}^{\prime}(t)>0 \quad \text { for } t \in\left(0, t_{u}\right) \quad \text { and } \quad h_{u}^{\prime}(t)<0 \quad \text { for } t>t_{u} .
$$

Similarly as in (3.3), it also follows that $h_{u}^{\prime}(t)=\frac{J(Q(t, u))}{t}$ for $t>0$. This gives (i) and (ii). Combining (4.3) with the fact that the map $X \backslash\{0\} \rightarrow \mathbb{R}, u \mapsto h_{u}^{\prime}(t)$ is continuous for fixed $t>0$, we also deduce that the map $X \backslash\{0\} \rightarrow(0, \infty), u \mapsto t_{u}$ is continuous, as claimed in (iii).

It thus remains to prove (iv). Let $u \in \mathcal{M}$ be an arbitrary minimizer for $I$ on $\mathcal{M}$. To show that $u$ is a critical point of $I$, we argue by contradiction and assume that there exists $v \in X$ such that $I^{\prime}(u) v<0$. Since $I$ is a $C^{1}$-functional on $X$, we may then fix $\varepsilon>0$ with the following property:

For every $\tau \in(0, \varepsilon)$, every $w \in X$ with $\|w\|_{X}<\varepsilon$ and every $\tilde{v} \in X$ with $\|\tilde{v}-v\|_{X}<\varepsilon$ we have

$$
I(u+w+\tau \tilde{v}) \leq I(u+w)-\varepsilon \tau .
$$

Using (iii) and the fact that $t_{u}=1$, we may then choose $\tau \in(0, \varepsilon)$ sufficiently small such that for $t^{\tau}:=t_{u+\tau v}$ we have

$$
\left|t^{\tau}-1\right|<\frac{\varepsilon}{\|u\|_{X}} \quad \text { and } \quad\left\|Q\left(t^{\tau}, v\right)-v\right\|_{X}<\varepsilon .
$$

Setting $w:=Q\left(t^{\tau}, u\right)-u$ and $\tilde{v}:=Q\left(t^{\tau}, v\right)$, we then have $\|w\|_{X}<\varepsilon$ and $\|\tilde{v}-v\|_{X}<\varepsilon$, so that, by the property above,

$$
\begin{aligned}
I\left(Q\left(t^{\tau}, u+\tau v\right)\right)=I\left(Q\left(t^{\tau}, u\right)+\tau Q\left(t^{\tau}, v\right)\right) & =I(u+w+\tau \tilde{v}) \leq I(u+w)-\varepsilon \tau \\
& <I(u+w)=I\left(Q\left(t^{\tau}, u\right)\right) \leq I(u)=c_{\mathcal{M}} .
\end{aligned}
$$

Since $Q\left(t^{\tau}, u+\tau v\right) \in \mathcal{M}$, this contradicts the definition of $c_{\mathcal{M}}$. Hence $u$ is a critical point of $I$.

Finally, to see that $u$ does not change sign, we note that $I(u)=I(|u|)$ and $J(u)=J(|u|)$, so that $|u|$ is a minimizer of $\left.I\right|_{\mathcal{M}}$ as well. Hence $|u|$ is a critical point of $I$ by the considerations above. By Remark 1.1, $|u| \in C^{2}\left(\mathbb{R}^{2}\right)$ and $-\Delta|u|+q|u|=0$ in $\mathbb{R}^{2}$ with some function $q \in L_{\text {loc }}^{\infty}\left(\mathbb{R}^{2}\right)$. Hence, the strong maximum principle and the fact that $u \neq 0$ implies that $|u|>0$ in $\mathbb{R}^{2}$, which shows that $u$ does not change sign.

Lemma 4.3. Let $p \geq 3$. For the energy values $c_{g}, c_{\mathcal{M}}, c_{m m}$ and $c_{m p}$ defined in the introduction, we then have $c_{g}=c_{\mathcal{M}}=c_{m m}=c_{m p}$.

Proof. By (1.14) we have $c_{\mathcal{M}} \leq c_{g} \leq c_{m p}$, and by Lemma 4.2 we have $c_{\mathcal{M}}=c_{m m}$. Moreover, from Lemma 2.6 we deduce that $c_{m p} \leq c_{m m}$. Thus the claim follows.

The proof of Theorem 1.2 is now completed by merely combining Theorem 1.1, Lemma 4.2 and Lemma 4.3 . 


\section{$5 \quad$ The symmetric setting}

This section is devoted to the proofs of Theorem 1.3 and Corollary 1.4. Some parts of the proof of Theorem 1.3 are similar to the proof of Theorem 1.1 and will therefore only be outlined. From now on, we fix a closed subgroup $G$ of the orthogonal group $O(2)$, and we let $\tau: G \rightarrow\{-1,1\}$ be a group homomorphism. We also consider the action $*$ of $G$ on $X$ defined by (1.17), and we assume that the corresponding invariant subspace

$$
X_{G}:=\{u \in X: A * u=u \text { for all } A \in G\}
$$

is not the null space. Our aim is to detect critical points of the restriction of the functional $I$ to $X_{G}$. By the principle of symmetric criticality (see [25, Theorem 1.28]), any critical point of the restriction of $I$ to $X_{G}$ (which we will denote by $I$ as well in the following) is a critical point of $I$.

From Lemmas 2.5 and 2.6, we easily deduce that

$$
0<m_{\rho} \leq c_{m p, G}:=\inf _{\gamma \in \Gamma_{G}} \max _{t \in[0,1]} I(\gamma(t))<\infty,
$$

where $\Gamma_{G}=\left\{\gamma \in C\left([0,1], X_{G}\right) \mid \gamma(0)=0, I(\gamma(1))<0\right\}$. Similar as in the proof of Lemma 3.2, we then deduce from Proposition 3.1 that there exists a sequence $\left\{u_{n}\right\} \subset X_{G}$ such that, as $n \rightarrow \infty$,

$$
I\left(u_{n}\right) \rightarrow c_{m p, G}, \quad\left\|I^{\prime}\left(u_{n}\right)\right\|_{X_{G}^{\prime}}\left(1+\left\|u_{n}\right\|_{X}\right) \rightarrow 0 \quad \text { and } \quad J\left(u_{n}\right) \rightarrow 0,
$$

where $J$ is defined in (1.11). The invariance of $I$ under the action $G$ implies that $I^{\prime}(v) w=0$ for all $v \in X_{G}$ and $w \in X_{G}^{\perp}$, where $X_{G}^{\perp}$ denotes the orthogonal complement of $X_{G}$ on $X$. Therefore, we have $\left\|I^{\prime}(v)\right\|_{X_{G}^{\prime}}=\left\|I^{\prime}(v)\right\|_{X^{\prime}}$ for all $v \in X_{G}$. Consequently, we may rewrite (5.2) as

$$
I\left(u_{n}\right) \rightarrow c_{m p, G}, \quad\left\|I^{\prime}\left(u_{n}\right)\right\|_{X^{\prime}}\left(1+\left\|u_{n}\right\|_{X}\right) \rightarrow 0 \quad \text { and } \quad J\left(u_{n}\right) \rightarrow 0 .
$$

We also have the following variant of Proposition 3.4. Here we consider the subspace

$$
\operatorname{Fix}(G):=\left\{x \in \mathbb{R}^{2}: A x=x \text { for all } A \in G\right\} \subset \mathbb{R}^{2} .
$$

Proposition 5.1. Let $p>2$, and let $\left(u_{n}\right)_{n}$ be a sequence in $X_{G}$ that satisfies

$$
c:=\sup _{n \in \mathbb{N}} I\left(u_{n}\right)<\infty \quad \text { and } \quad\left\|I^{\prime}\left(u_{n}\right)\right\|_{X^{\prime}}\left(1+\left\|u_{n}\right\|_{X}\right) \rightarrow 0, \quad J\left(u_{n}\right) \rightarrow 0 \quad \text { as } n \rightarrow \infty .
$$

Then, after passing to a subsequence, one of the following occurs:

(I) $\left\|u_{n}\right\| \rightarrow 0$ and $I\left(u_{n}\right) \rightarrow 0$ as $n \rightarrow \infty$.

(II) There exist points $y_{n} \in \operatorname{Fix}(G), n \in \mathbb{N}$ such that

$$
y_{n} * u_{n} \rightarrow u \quad \text { strongly in } X \text { as } n \rightarrow \infty
$$

for some nonzero critical point $u \in X_{G}$ of $I$. 
Proof. Suppose that $(I)$ does not hold. By Proposition 3.4, we may pass to a subsequence such that

$$
\tilde{y}_{n} * u_{n} \rightarrow \tilde{u} \quad \text { in } X \text { as } n \rightarrow \infty
$$

for suitable points $\tilde{y}_{n} \in \mathbb{R}^{2}, n \in \mathbb{N}$ and some nonzero critical point $\tilde{u} \in X$ of $I$. We first claim that

$$
\sup \left\{\left|A \tilde{y}_{n}-\tilde{y}_{n}\right|: A \in G, n \in \mathbb{N}\right\}<\infty .
$$

To see this, we set $z_{n}:=-\tilde{y}_{n}$ for $n \in \mathbb{N}$, and we let $\left(A_{n}\right)_{n} \subset G$ be an arbitrary sequence. Since $A_{n} * u_{n}=u_{n}$ for every $n \in \mathbb{N}$, we have

$$
\begin{array}{r}
\left|A_{n} *\left(z_{n} * \tilde{u}\right)-z_{n} * \tilde{u}\right|_{2} \leq\left|A_{n} *\left(z_{n} * \tilde{u}-u_{n}\right)\right|_{2}+\left|u_{n}-z_{n} * \tilde{u}\right|_{2}=2\left|z_{n} * \tilde{u}-u_{n}\right|_{2} \\
=2\left|\tilde{u}-\tilde{y}_{n} * u_{n}\right|_{2} \rightarrow 0 \quad \text { as } n \rightarrow \infty .
\end{array}
$$

Setting $v_{n}:=A_{n} * \tilde{u}$ and $\zeta_{n}:=A_{n} z_{n}-z_{n}$ for $n \in \mathbb{N}$, we also find that

$$
\begin{array}{r}
\left|A_{n} *\left(z_{n} * \tilde{u}\right)-z_{n} * \tilde{u}\right|_{2}^{2}-2|\tilde{u}|_{2}^{2}=-2 \tau\left(A_{n}\right) \int_{\mathbb{R}^{2}} \tilde{u}\left(A_{n}^{-1} x-z_{n}\right) \tilde{u}\left(x-z_{n}\right) d x \\
=-2 \tau\left(A_{n}\right) \int_{\mathbb{R}^{2}} \tilde{u}\left(A_{n}^{-1}\left(y-\zeta_{n}\right)\right) \tilde{u}(y) d y=-2 \int_{\mathbb{R}^{2}}\left(\zeta_{n} * v_{n}\right) \tilde{u} d y
\end{array}
$$

Since $G$ is compact as a closed subgroup of $O(2)$, we may pass to a subsequence such that $A_{n} \rightarrow$ $A \in G$ as $n \rightarrow \infty$, which implies that $v_{n} \rightarrow v:=A * \tilde{u}$ in $X$ and therefore

$$
\left|\zeta_{n} *\left(v_{n}-v\right)\right|_{2}=\left|v_{n}-v\right|_{2} \rightarrow 0 \quad \text { as } n \rightarrow \infty .
$$

Combining (5.6), (5.7) and (5.8) gives

$$
\lim _{n \rightarrow \infty} \int_{\mathbb{R}^{2}}\left(\zeta_{n} * v\right) \tilde{u} d z=|\tilde{u}|_{2}^{2}>0
$$

From this we deduce that $\left(\zeta_{n}\right)_{n}$ remains bounded, since otherwise $\zeta_{n} * v \rightarrow 0$ in $L^{2}\left(\mathbb{R}^{2}\right)$ after passing to a subsequence. Since

$$
\left|A_{n} \tilde{y}_{n}-\tilde{y}_{n}\right|=\left|A_{n} z_{n}-z_{n}\right|=\left|\zeta_{n}\right| \quad \text { for } n \in \mathbb{N}
$$

we thus infer that also $A_{n} \tilde{y}_{n}-\tilde{y}_{n}$ remains bounded, and this gives (5.5).

We now replace $\tilde{y}_{n}$ by

$$
y_{n}:=\frac{1}{\mu(G)} \int_{G} A \tilde{y}_{n} d \mu(A) \in \operatorname{Fix}(G), \quad n \in \mathbb{N},
$$

where $\mu$ denotes the Haar measure of $G$. By (5.5) we infer that $y_{n}-\tilde{y}_{n}$ remains bounded in $\mathbb{R}^{2}$ as $n \rightarrow \infty$, and thus we may pass to a subsequence such that $y_{n}-\tilde{y}_{n} \rightarrow r \in \mathbb{R}^{2}$. Consequently,

$$
y_{n} * u_{n} \rightarrow u:=r * \tilde{u} \quad \text { in } X \text { as } n \rightarrow \infty
$$

Finally, for $A \in G$ and $x \in \mathbb{R}^{2}$ we have, since $y_{n} \in \operatorname{Fix}(G)$,

$$
\begin{aligned}
{[A * u](x) } & =\lim _{n \rightarrow \infty} A *\left(y_{n} * u_{n}\right)=\tau(A) \lim _{n \rightarrow \infty} u_{n}\left(A^{-1} x-y_{n}\right)=\tau(A) \lim _{n \rightarrow \infty} u_{n}\left(A^{-1}\left(x-y_{n}\right)\right) \\
& =\lim _{n \rightarrow \infty}\left[y_{n} *\left(A * u_{n}\right)\right](x)=\lim _{n \rightarrow \infty}\left[y_{n} * u_{n}\right](x)=u(x) .
\end{aligned}
$$

We thus conclude that $u \in X_{G}$. This shows that alternative $(I I)$ holds, as claimed. 
Proof of Theorem 1.3 (completed). Using Proposition 5.1, we may now complete the proof of Theorem 1.3 precisely as we completed the proof of Theorem 1.1 in the last part of Section 3 , replacing $X$ by $X_{G}$ and $c_{m p}$ by $c_{m p, G}$.

It thus remains to give the proof of Corollary 1.4 .

Proof of Corollary 1.4. Similarly as in Example1.1(iii) we consider, for $n \in \mathbb{N}$, the subgroup $G_{n}$ of $O(2)$ of order $2 \cdot 3^{n}$ generated by the (counter-clockwise) $\frac{\pi}{3^{n}}$-rotation

$$
A_{n} \in O(2), \quad A_{n} x=\left(x_{1} \cos \frac{\pi}{3^{n}}-x_{2} \sin \frac{\pi}{3^{n}}, x_{1} \sin \frac{\pi}{3^{n}}+x_{2} \cos \frac{\pi}{3^{n}}\right) \quad \text { for } x=\left(x_{1}, x_{2}\right) \in \mathbb{R}^{2} .
$$

Let $\tau_{n}: G_{n} \rightarrow\{-1,1\}$ be the homomorphism defined by

$$
\tau_{n}\left(A_{n}^{j}\right)=(-1)^{j} \quad \text { for } j=1, \cdots, 2 \cdot 3^{n},
$$

where $A_{n}^{j}$ is the $\frac{j \pi}{3^{n}}$-rotation. By definition, we have that

$$
X_{G_{n+1}} \subset X_{G_{n}} \quad \text { for } n \in \mathbb{N} \text {. }
$$

Consequently, we also have that

$$
c_{m p, G_{n+1}} \geq c_{m p, G_{n}} \geq c_{m p, G_{1}}>0 \quad \text { for all } n \in \mathbb{N} .
$$

Then Theorem 1.3 applies and yields a nonradial sign-changing solution $u_{n} \in X_{G_{n}}$ with $I\left(u_{n}\right)=$ $c_{m p, G_{n}}$ for every $n \in \mathbb{N}$. Suppose by contradiction that

$$
\limsup _{n \rightarrow \infty} I\left(u_{n}\right)<\infty
$$

Applying Proposition 5.1 for fixed $n \in \mathbb{N}$ and using the fact that $\operatorname{Fix}\left(G_{n}\right)=\{0\}$ for all $n \in \mathbb{N}$, we may now pass to a subsequence such that

$$
u_{n} \rightarrow u \quad \text { in } X
$$

where

$$
u \in \bigcap_{n \in \mathbb{N}} X_{G_{n}}
$$

and $u \in X \backslash\{0\}$ is a critical point of $I$. As noted in Remark 1.1, it then follows that $u$ is of class $C^{2}$ on $\mathbb{R}^{2}$. From (5.10) we then deduce that

$$
u(x)=-u\left(A_{n} x\right) \quad \text { for all } x \in \mathbb{R}^{2}, n \in \mathbb{N} .
$$

Since $A_{n} x \rightarrow x$ for $x \in \mathbb{R}^{2}$ as $n \rightarrow \infty$, it follows that $u(x)=0$ for $x \in \mathbb{R}^{2}$. This is a contradiction, and thus the claim follows.

\section{Acknowledgements}

This work was completed when the first author visited Goethe-University of Frankfurt in 20162017, and he would like to thank Tobias Weth for his hospitality. The first author was partially supported by Natural Science Foundation of China (Nos. 11171135, 51276081, 11571140, 11671077, 11601204) and Major Project of Natural Science Foundation of Jiangsu Province Colleges and Universities (No. 14KJA100001). 


\section{References}

[1] A. Ambrosetti, D. Ruiz, Multiple bound states for the Schrödinger-Poisson equation, Commun. Contemp. Math. 10 (2008), 1-14.

[2] A. Azzollini, A. Pomponio, Ground state solutions for the nonlinear Schrödinger-Maxwell equations, J. Math. Anal. Appl. 345 (2008), 90-108.

[3] J. Bellazzini, L. Jeanjean, T. Luo, Existence and instability of standing waves with prescribed norm for a class of Schrödinger-Poisson equations, Proc. Lond. Math. Soc. (2013), 303-339.

[4] H. Berestycki, P.L. Lions, Nonlinear scalar field equations I, Arch. Ration. Mech. Anal. 82 (1983), 313-346.

[5] G. Cerami, G. Vaira, Positive solutions for some non autonomous Schrödinger-Poisson systems, J. Differential Equations 248 (2010), 521-543.

[6] S. Cingolani, T. Weth, On the planar Schrödinger-Poisson system, Ann. I. H. Poincaré-AN 33 (2016), 169-197.

[7] M.J. Esteban, P.L. Lions, Existence and nonexistence results for semilinear elliptic problems in unbounded domains, Proc. Roy. Soc. Edinburgh Sect. A 93 (1982/83), 1-14.

[8] T. D'Aprile, D. Mugnai, Solitary waves for nonlinear Klein-Gordon-Maxwell and SchrödingerMaxwell equations, Proc. Roy. Soc. Edinburgh Sect. A 134 (2004), 893-906.

[9] T. D'Aprile, D. Mugnai, Non-existence results for the coupled Klein-Cordon-Maxwell equations, Adv. Nonlinear Stud. 4 (2004), 307-322.

[10] T. D'Aprile, J.C. Wei, On bound states concentrating on spheres for the Maxwell-Schrödinger equation, SIAM J. Math. Anal. 37 (2005), 321-342.

[11] J. Hirata, N. Ikoma, K. Tanaka, Nonlinear scalar field equations in $\mathbb{R}^{N}$ : mountain pass and symmetric mountain pass approaches, Topol. Methods Nonlinear Anal. 35 (2010), 253-276.

[12] L. Jeanjean, Existence of solutions with prescribed norm for semilinear elliptic equations, Nonlinear Anal. 28 (1997), 1633-1659.

[13] G.B. Li, C. Wang, The existence of a nontrivial solution to a nonlinear elliptic problem of linking type without the Ambrosetti-Rabinowitz condition, Ann. Acad. Sci. Fenn. Math. 36 (2011), 461-480.

[14] E.H. Lieb, Sharp constants in the Hardy-Littlewood-Sobolev and related inequalities, Ann. Math. 118 (1983), 349-374.

[15] P.L. Lions, The concentration-compactness principle in the calculus of variations. The locally compact case, part I, Ann. Inst. H. Poincaré Anal. Non Linéaire 1 (1984), 109-145.

[16] N.J. Mauser, The Schrödinger-Poisson- $X_{\alpha}$ equation, Appl. Math. Lett. 14 (2001), 759-763. 
[17] V. Moroz, J. Van Schaftingen, Existence of groundstates for a class of nonlinear Choquard equations, Trans. Amer. Math. Soc. 367 (2015), 6557-6579.

[18] S.I. Pohozaev, On the eigenfunctions of the equation $\Delta u+\lambda f(u)=0$, Soviet Math. Dokl. 5 (1965), 1408-1411.

[19] P.H. Rabinowitz, On a class of nonlinear Schrödinger equations, Z. Angew. Math. Phys. 43 (1992), 270-291.

[20] D. Ruiz, The Schrödinger-Poisson equation under the effect of a nonlinear local term, J. Funct. Anal. 237 (2006), 655-674.

[21] J. Stubbe, Bound states of two-dimensional Schrödinger-Newton equations, arXiv: 0807.4059 -v1, 2008.

[22] A. Szulkin, T. Weth, The method of Nehari manifold, in: D.Y. Gao, D. Motreanu (Eds.), Handbook of Nonconvex Analysis and Applications, International Press, Boston, 2010, pp. 597-632.

[23] J. Wang, J.X. Xu, F.B. Zhang, X.M. Chen, Existence and concentration of positive ground state solutions for semilinear Schrödinger-Poisson systems in $\mathbb{R}^{3}$, Calc. Var. Partical Differential Equations. 48 (2013), 243-273.

[24] Z.P. Wang, H.S. Zhou, Positive solution for a nonlinear stationary Schrödinger-Poisson system in $\mathbb{R}^{3}$, Discrete Contin. Dyn. Syst. 18 (2007), 809-816.

[25] M. Willem, Minimax Theorems, Birkhäuser, Boston, 1996.

[26] L.G. Zhao, H.D. Liu, F.K. Zhao, Existence and concentration of solutions for the SchrödingerPoisson equations with steep well potential, J. Differential Equations 255 (2013), 1-23.

[27] L.G. Zhao, F.K. Zhao, On the existence of solutions for the Schröinger-Poisson equations, J. Math. Anal. Appl. 346 (2008), 155-169. 